\title{
Generation of dark radiation in the bulk inflaton model
}

$\operatorname{AUTHOR}(\mathrm{S}):$

Tanaka, T; Himemoto, Y

CITATION:

Tanaka, T ... [et al]. Generation of dark radiation in the bulk inflaton model. PHYSICAL REVIEW D 2003, 67(10): 104007.

ISSUE DATE:

2003-05-15

URL:

http://hdl.handle.net/2433/49998

RIGHT:

Copyright 2003 American Physical Society 
PHYSICAL REVIEW D 67, 104007 (2003)

\title{
Generation of dark radiation in the bulk inflaton model
}

\author{
Takahiro Tanaka \\ Yukawa Institute for Theoretical Physics, Kyoto University, Kyoto 606-8502, Japan \\ Yoshiaki Himemoto \\ Yukawa Institute for Theoretical Physics, Kyoto University, Kyoto 606-8502, Japan \\ and Department of Earth and Space Science, Graduate School of Science, Osaka University, Toyonaka 560-0043, Japan
}

(Received 15 January 2003; published 14 May 2003)

\begin{abstract}
We investigate the dynamics of a bulk scalar field with various decay channels in the Randall-Sundrum infinite braneworld scenario. A bulk scalar field in this scenario has a quasilocalized mode which dominates the late-time behavior near the brane. As for this mode, an interesting point is the presence of dissipation caused by the escape of the energy in the direction away from the brane, even if the bulk scalar field does not have the interaction with the other bulk fields in the bulk and fields on the brane. We can interpret that this lost energy is transferred to the dark radiation. We show that such an effective 4-dimensional description for a bulk scalar field is valid including the various processes of energy dissipation.
\end{abstract}

DOI: 10.1103/PhysRevD.67.104007

PACS number(s): $04.50 .+\mathrm{h}, 98.80 . \mathrm{Cq}$

\section{INTRODUCTION}

The braneworld scenario has provided a new scheme to derive 4-dimensional effective theory from a fundamental higher dimensional one [1]. In particular, the second model proposed by Randall and Sundrum (RS2) has demonstrated an exciting possibility that the extra dimensions may extend infinitely [2]. A lot of work has been done on various aspects of this model. Extensive research based on relativity and cosmology, which is closely related to the issue we will discuss in this paper, has also been developed [3-6].

The background spacetime of the original RS model is given by a $Z_{2}$-symmetric solution of the 5-dimensional Einstein equations with a negative cosmological constant. However, in the framework of string theory, it is more likely that there are various fields other than the graviton in the 5-dimensional bulk. Thus, the dynamics of a minimally coupled 5-dimensional massive scalar field in the braneworld scenario of RS2 type has been studied, and it was shown that a bulk scalar field can play the role of inflaton field [7-9]. We refer to such a model as the bulk inflaton model.

Prior to the proposal of the bulk inflaton model, Dubovsky, Rubakov, and Tinyakov have analyzed the dynamics of a bulk scalar field with the mass $m$ on the RS2 Minkowski brane background [10]. They have found that the bulk scalar field has a quasilocalized mode which dominates the late-time behavior near the brane. When this mode dominates, the scalar field seen on the brane oscillates with the frequency given by $m / \sqrt{2}$ and decays gradually. Besides this interesting intrinsic decay, when we try to construct a more realistic bulk inflaton model, we also need to take account of the dissipation due to the interaction with other bulk fields and fields on the brane. ${ }^{1}$ We discuss such decay processes in this paper, focusing on the effect on the cosmic expansion law realized on the brane. Related discussions about the interaction between the bulk and the fields on the brane were

\footnotetext{
${ }^{1}$ The latter process was investigated in our previous paper [9].
}

given in [11]. In particular, generation of dark radiation via collision of particles on the brane was discussed in [12].

This paper is organized as follows. In Sec. II we will review the general formulas for the cosmic expansion law under the presence of bulk fields. In Sec. III we will study various decay channels of the bulk scalar field, and show how the decay products affect the cosmic expansion law on the brane. Section IV is devoted to summary.

\section{COSMIC EXPANSION LAW IN THE GENERALIZED RANDALL-SUNDRUM BRANEWORLD}

We consider the braneworld scenario of the RS2 type, starting with the 5-dimensional Einstein equations

$$
R_{a b}-\frac{1}{2} g_{a b} R+\Lambda_{5} g_{a b}=\kappa_{5}^{2}\left[T_{a b}+S_{a b} \delta\left(r-r_{0}\right)\right],
$$

where $r$ is the proper coordinate normal to the brane, and the brane is located at $r=r_{0}$ at the fixed point of the $Z_{2}$ symmetry. $S_{a b}$ represents the energy-momentum tensor composed of the brane tension $\sigma$ and the contribution of matter fields confined on the brane $\tau_{a b}$ :

$$
S_{a b}=-\sigma q_{a b}+\tau_{a b}
$$

where $q_{a b}$ is the metric induced on the brane. We choose $\sigma$ $:=\sqrt{-6 \Lambda_{5}} / \kappa_{5}^{2}=6 / \kappa_{5}^{2} \ell$ so that the Randall-Sundrum flat brane is realized as the ground state. Here $\ell$ is the bulk curvature radius. $T_{a b}$ is the effective 5-dimensional energymomentum tensor of the bulk fields. Here we do not specify the form of $T_{a b}$.

It is now well known that the 4-dimensional effective Einstein equation is derived from Eq. (1) as [3]

$$
{ }^{(4)} G_{\mu \nu}=\kappa_{4}^{2}\left(\tau_{\mu \nu}+\tau_{\mu \nu}^{(\pi)}+\tau_{\mu \nu}^{(B)}+\tau_{\mu \nu}^{(E)}\right),
$$

where $\kappa_{4}^{2}=\kappa_{5}^{2} / \ell$ and 


$$
\begin{aligned}
& \tau_{\mu \nu}^{(\pi)}=-\frac{\kappa_{5}^{2} \ell}{24}\left[6 \tau_{\mu \alpha} \tau_{\nu}^{\alpha}-2 \tau \tau_{\mu \nu}-q_{\mu \nu}\left(3 \tau_{\alpha \beta} \tau^{\alpha \beta}-\tau^{2}\right)\right], \\
& \tau_{\mu \nu}^{(B)}=\frac{2 \ell}{3}\left[T_{a b} q_{\mu}^{a} q_{\nu}^{b}+q_{\mu \nu}\left(T_{a b} n^{a} n^{b}-\frac{1}{4} T_{a}^{a}\right)\right]_{r=r_{0}}, \\
& \tau_{\mu \nu}^{(E)}=-\frac{\ell}{\kappa_{5}^{2}}\left[{ }^{(5)} C_{a b c d} n^{a} n^{c} q_{\mu}^{b} q_{\nu}^{d}\right]_{r=r_{0}},
\end{aligned}
$$

where $n^{a}$ is the unit vector pointing outward and normal to the brane. Here $\tau_{\mu \nu}^{(E)}$ is the energy-momentum tensor related to the 5-dimensional Weyl tensor ${ }^{(5)} C_{a b c d}$.

To discuss the braneworld cosmology, we assume the spatial homogeneity and isotropy for $q_{\mu \nu}$ and for each component of the energy-momentum tensor. We define the energy density of the 4-dimensional ordinary matter fields by $\rho$ $:=u^{\mu} u^{\nu} \tau_{\mu \nu}$, where $u^{\mu}$ is the timelike unit vector associated with comoving observers on the brane. Similarly, we define $\rho^{(i)}:=u^{\mu} u^{\nu} \tau_{\mu \nu}^{(i)}$ for each component. Then, Eq. (3) gives the Friedmann equation on the brane:

$$
H^{2}=\frac{\kappa_{4}^{2}}{3}\left[\left(1+\frac{\rho}{2 \sigma}\right) \rho+\rho^{(B)}+\rho^{(E)}\right],
$$

where $H$ is the Hubble parameter on the brane. Here we have used the fact $\rho^{(\pi)}=\rho^{2} / 2 \sigma$.

Next we consider the equations for the evolution of the energy density. From the 5-dimensional conservation law integrated over infinitesimally thin layer near the brane, the evolution equation for $\rho$ is obtained as

$$
\dot{\rho}+4 H \rho+H \tau_{\mu}^{\mu}=\left.2 T_{a b} u^{a} n^{b}\right|_{r=r_{0}},
$$

where an overdot means differentiation with respect to the cosmic proper time $t$.

On the other hand, 4-dimensional Bianchi identity implies

$$
\dot{\rho}^{(\text {tot })}+4 H \rho^{(\text {tot })}+H \tau_{\mu}^{(\text {tot }) \mu}=0,
$$

where we have defined $\rho^{(\mathrm{tot})}:=\rho+\rho^{(\pi)}+\rho^{(B)}+\rho^{(E)}$ and $\tau_{\mu \nu}^{(\text {tot })}$ in the same way. Then, using Eq. (6) and the facts that $\tau_{\mu}^{(\pi) \mu}=(\rho / \sigma)\left(\tau_{\mu}^{\mu}+2 \rho\right), \tau_{\mu}^{(B) \mu}=2 \ell T_{a b} n^{a} n^{b}$, and $\tau_{\mu}^{(E) \mu}$ $=0$, we find that the effective energy density of the bulk field $\rho_{\text {eff }}:=\rho^{(B)}+\rho^{(E)}$ satisfies

$$
\dot{\rho}_{\mathrm{eff}}+4 H \rho_{\mathrm{eff}}=\left[-2\left(1+\frac{\rho}{\sigma}\right) T_{a b} u^{a} n^{b}-2 H \ell T_{a b} n^{a} n^{b}\right]_{r=r_{0}} .
$$

The above Eqs. (5), (6), and (8) completely determine the cosmic expansion law once we know the evolution of the energy-momentum tensor of the 5-dimensional fields evaluated on the brane.

\section{DISSIPATION OF THE BULK SCALAR FIELD}

As a phenomenological model of a bulk scalar field with dissipation, we consider the field equation given by

$$
\square_{5} \phi-m^{2} \phi=\left(\Gamma_{d} \ell \delta\left(r-r_{0}\right)+\frac{\hat{\Gamma}_{b}(r)}{N(r)}\right) \dot{\phi},
$$

where $m^{2}$ is the mass squared of a bulk scalar field and $N(r)$ is the lapse function. Here $\Gamma_{d}$ represents the rate of the decay on the brane, while $\hat{\Gamma}_{b}(r)$ is a function specifying the rate of energy release to the bulk. We call this $\phi$ field the bulk inflaton, although our main interest is not in the period of inflation but in the period of reheating after inflation. For a moment, we do not specify the functional form of $\hat{\Gamma}_{b}(r)$. In this setting, we study the cosmic expansion law by analyzing the set of equations (5), (6), and (8).

\section{A. Dark radiation from escaping bulk inflaton}

First, we consider the simplest case in which there is no bulk field other than the bulk inflaton field $\phi$ that has no direct interaction to the fields on the brane. Namely, $T_{a b}$ $=T_{a b}^{(\phi)}$ and $\Gamma_{d}=\hat{\Gamma}_{b}=0$, where

$$
T_{a b}^{(\phi)}=\phi_{, a} \phi_{, b}-g_{a b}\left(\frac{1}{2} g^{c d} \phi_{, c} \phi_{, d}+\frac{1}{2} m^{2} \phi^{2}\right)
$$

is the energy-momentum tensor of the bulk inflaton field. Examining this simplest case is instructive to figure out an important difference between a bulk field and a field on the brane.

According to the analyses of the dynamics of a massive bulk scalar field on the RS2 flat brane background [10] and on the de Sitter brane background [7], there is a quasilocalized mode which dominates the late-time behavior. This quasilocalized mode corresponds to a pair of the first poles appearing in the retarded Green function in the frequency domain. To summarize the results obtained in the above references, $\phi$ behaves as $\propto e^{[ \pm i \omega-(3 H / 2)-(\Gamma / 2)] t}$ with

$$
\begin{aligned}
& \omega \approx \sqrt{m_{\mathrm{eff}}^{2}-\frac{9}{4} H^{2}} \underset{H^{2} / m^{2} \rightarrow 0}{\longrightarrow} m_{\mathrm{eff}}, \\
& \Gamma=\Gamma_{\mathrm{esc}} \approx \frac{m^{2}\left(m^{2}-4 H^{2}\right) \ell^{4}}{8 \omega} \\
& \times \operatorname{Im}\left[\psi\left(\frac{1}{2}-\frac{i \omega}{H}\right)\right] \underset{H^{2} / m^{2} \rightarrow 0}{\longrightarrow} m^{3} \ell^{2} \pi / 8 \sqrt{2},
\end{aligned}
$$

under the conditions

$$
m \ell \text { and } H \ell \ll 1 \text {. }
$$

Here $m_{\text {eff }}^{2}=m^{2} / 2$, and $\psi(z) \equiv \Gamma^{\prime}(z) / \Gamma(z)$ is the poly-Gamma function. The bulk inflaton oscillates with the frequency $\omega$ and decays intrinsically even if we do not introduce dissipation effects due to the coupling to other fields. From the 5-dimensional point of view, this decay of the amplitude of oscillation occurs as a result of the escape of the energy in the direction away from the brane. We note that the above expressions are valid only when $m_{\text {eff }}^{2} / H^{2}>\frac{9}{4}$. Here our discussion is therefore restricted to the reheating era after inflation.

Let us consider the effective energy density of the bulk inflaton field from the viewpoint of 4-dimensional observers. In the present case, a $Z_{2}$-symmetric boundary condition im- 
plies $\phi_{, a} n^{a}=0$. Therefore we have $T_{a b}^{(\phi)} u^{a} n^{b}=0$, and then the energy conservation equation (6) reduces to the same equation as in the standard 4-dimensional cosmology:

$$
\dot{\rho}+4 H \rho+H \tau_{\mu}^{\mu}=0 .
$$

Furthermore, from Eq. (8), we find that $\rho_{\text {eff }}=\rho^{(E)}+\rho^{(\phi)}$ satisfies

$$
\dot{\rho}_{\mathrm{eff}}+4 H \rho_{\mathrm{eff}}=-H \ell\left[\dot{\phi}^{2}-m^{2} \phi^{2}\right]_{r=r_{0}} .
$$

This equation tells us that the evolution of $\rho_{\text {eff }}$ depends on the evolution of $\phi$ on the brane. Therefore, in order to determine the cosmic expansion law, we need to know the evolution of the bulk scalar field.

Next, for comparison we propose a 4-dimensional model composed of a scalar field $\Phi$ and the dark radiation in addition to the ordinary matter fields. We should stress that in the 4-dimensional model the dark radiation simply means a component of energy density decoupled from the ordinary matter fields. As for the ordinary matter fields, we use the same notations as in the case of the 5-dimensional model. Then the conservation law for the ordinary matter fields is identical to Eq. (13).

We define a scalar field $\Phi$ which reproduces the late-time behavior of the value of $\phi$ on the brane besides an overall normalization factor. We assume that $\Phi$ has mass $m_{\text {eff }}$ and decays only into the dark radiation with the decay width $\Gamma_{\text {esc }} / 2$. The equation of motion for the homogeneous part of the scalar field is given by

$$
\ddot{\Phi}+\left(3 H+\Gamma_{\text {esc }}\right) \dot{\Phi}+m_{\text {eff }}^{2} \Phi=0 .
$$

Using $\Gamma_{\text {esc }} \ll m$, which follows from $m \ell \ll 1$, it is easy to see that $\Phi$ also behaves as $\propto e^{\left[ \pm i \omega-(3 H / 2)-\left(\Gamma_{\text {esc }} / 2\right)\right] t}$ with the same $\omega$ in Eq. (11).

The Hubble equation is given by $H^{2}=\left(\kappa_{4}^{2} / 3\right)\left(\rho+\tilde{\rho}_{\text {eff }}\right)$, with $\tilde{\rho}_{\text {eff }}:=\rho^{(\Phi)}+\rho^{(D)}$, where $\rho^{(\Phi)}$ and $\rho^{(D)}$ are the energy density of the $\Phi$ field and the dark radiation, respectively. Since the scalar field $\Phi$ and the dark radiation are decoupled from the ordinary matter fields by assumption, they separately satisfy the conservation law. The conservation law for $\Phi$ and the dark radiation reduces to

$$
\dot{\tilde{\rho}}_{\mathrm{eff}}+4 H \tilde{\rho}_{\mathrm{eff}}=-H \tau_{\mu}^{(\Phi) \mu}=-H\left(\dot{\Phi}^{2}-2 m_{\mathrm{eff}}^{2} \Phi^{2}\right) .
$$

Then, with simple identification

$$
\Phi=\sqrt{\ell} \phi
$$

it is easy to see that the 4-dimensional and the 5-dimensional models presented above realize the same cosmic expansion in the law energy regime (12), in which the contribution from $\rho^{(\pi)}$ is neglected.

From the viewpoint of the 4-dimensional model, we can easily write down the evolution of the energy density of $\Phi$ field as

$$
\dot{\rho}^{(\Phi)}+4 H \rho^{(\Phi)}+H \tau_{\mu}^{(\Phi) \mu}=-\Gamma_{\mathrm{esc}} \dot{\Phi}^{2}
$$

Then, with the aid of Eq. (16), we have

$$
\dot{\rho}^{(D)}+4 H \rho^{(D)}=\Gamma_{\mathrm{esc}} \dot{\Phi}^{2}
$$

Thus, we can naturally interpret that the $\Phi$ field decays into the dark radiation. Therefore, from the correspondence between the 4-dimensional and the 5-dimensional models, we are led to the interpretation that the dark radiation is generated as a result of this decay process in the 5-dimensional model.

Usually, when we consider decay process, it leaves some decay products. However, the present decay process of the bulk inflaton apparently does not have corresponding decay products, since we do not consider the interaction with the other fields. Then, one may think that once the bulk field decays in this manner, its effective energy density is simply lost without being transferred into another form. As was expected from the energy conservation law, this naive speculation is incorrect. In fact, the lost energy was shown to be transferred into the dark radiation component.

Before closing this section, we mention that $\rho^{(\Phi)}$ in the 4-dimensional model does not correspond to $\rho^{(\phi)}=\rho^{(B)}$ in the 5-dimensional model. Hence, neither does $\rho^{(D)}$ to $\rho^{(E)}$ since $\rho^{(\Phi)}+\rho^{(D)}=\tilde{\rho}_{\text {eff }}=\rho_{\text {eff }}=\rho^{(\phi)}+\rho^{(E)}$. The fact that $\rho^{(\Phi)}$ $\neq \rho^{(\phi)}$ will be easily seen by explicitly evaluating $\rho^{(B)}$ by using Eqs. (4) and (10) as $\rho^{(\phi)}=(\ell / 4)\left[\dot{\phi}^{2}+m^{2} \phi^{2}\right]$ $=(1 / 4) \dot{\Phi}^{2}+\left(m_{\mathrm{eff}}^{2} / 2\right) \Phi^{2}$. This expression shows that $\rho^{(\Phi)}$ $=\rho^{(\phi)}+\ell \dot{\phi}^{2} / 4$.

\section{B. Decay of the bulk scalar field to other fields}

In this section, we analyze the field equation (9) without neglecting the dissipation terms on the background of the 5-dimensional anti-de Sitter space with a boundary de Sitter brane. We make use of the fact that the late-time behavior is determined by the singularities in the retarded Green function in the frequency domain, as was done in Ref. [9].

The background metric can be written as

$$
d s^{2}=R(y)^{2}\left(d y^{2}-d \tau^{2}+e^{2 \tau} d x_{(3)}^{2}\right),
$$

where $R(y) \equiv \ell \sinh ^{-1}\left(|y|+y_{0}\right)$. The brane is located at $y$ $=0$, and $y_{0}$ is specified by $\sinh y_{0}=H \ell$. The proper cosmological time on the brane $t$ is related to $\tau$ by $\tau \equiv H t$. Setting $\phi=R^{-3 / 2} e^{-3 \tau / 2} \Psi(y, \tau)$, Eq. (9) reduces to

$$
\left[-\partial_{\tau}^{2}+\partial_{y}^{2}-W(y)-U(y) \partial_{\tau}\right] \Psi=0
$$

with

$$
W(y)=\frac{15+4 m^{2} \ell^{2}}{4 \sinh ^{2}\left(|y|+y_{0}\right)}-\frac{3 \sqrt{1+H^{2} \ell^{2}}}{H \ell} \delta(y)-\frac{3}{2} U(y),
$$

$U(y)=\Gamma_{d} \ell \delta(y)+\hat{\Gamma}_{b}(y) R(y)$. 
According to Ref. [9], the poles of the retarded Green function correspond to the solutions of Eq. (21) that satisfy the outgoing boundary condition. The solutions can be found in the form of

$$
\Psi_{p} \propto e^{-i p \tau} u_{p}
$$

Then, Eq. (21) reduces to an eigenvalue equation for $u_{p}$ having $p^{2}$ as the eigenvalue. When $\hat{\Gamma}_{b}=0$, the equation for $u_{p}$ can be solved with the outgoing boundary condition as

$$
u_{p}(y)=\frac{1}{\mathcal{N}} P_{\nu-1 / 2}^{i p}\left[\operatorname{coth}\left(|y|+y_{0}\right)\right] .
$$

where $\nu:=\sqrt{m^{2} \ell^{2}+4}$. The normalization constant $\mathcal{N}$ is to be chosen so as to satisfy $\int_{-\infty}^{\infty} d y\left|u_{p}\right|^{2}=1$. Here we have not yet imposed the boundary condition on the brane. The condition of the $Z_{2}$ symmetry,

$$
\left[\left\{\partial_{y}+\frac{3}{2} \frac{\sqrt{1+H^{2} \ell^{2}}}{H \ell}+\left(i p+\frac{3}{2}\right) \frac{\Gamma_{d} \ell}{2}\right\} u_{p}(y)\right]_{y=0+}=0,
$$

gives the equation that determines the eigenvalue, i.e., the location of the poles in the retarded Green function. We focus on the eigenmode with the eigenvalue $p$ that has the largest imaginary part in order to discuss the late-time behavior of the bulk inflaton field. Under the conditions (12), Eq. (26) for this eigenmode reduces to a quadratic equation as

$$
p^{2}+\left(i p+\frac{3}{2}\right) \frac{\Gamma_{d}}{H}+\frac{9}{4}-\frac{m^{2}}{2 H^{2}} \approx 0 .
$$

In order to illustrate the effect due to the dissipation in the bulk, we consider two specific analytically solvable examples given by

$$
\begin{aligned}
& \hat{\Gamma}_{b}(y) R(y)=\Gamma_{b} H^{-1} \quad[\text { case (I)], } \\
& \hat{\Gamma}_{b}(y) R(y)=2 \Gamma_{b} H R(y)^{2}, \quad[\text { case (II)], }
\end{aligned}
$$

where $\Gamma_{b}$ is a constant. In case (I) the first term $p^{2}$ in Eq. (27) is replaced with $p^{2}+(i p+3 / 2) \Gamma_{b} H^{-1}$, while in case (II) $m^{2}$ in the same equation is replaced with $m^{2}-2$ (ip $+3 / 2) \Gamma_{b} H$. Then, in both cases we have the same equation to determine the eigenvalue of the largest imaginary part, and we find that a pair of such eigenvalues is given by

$$
p_{ \pm} \approx \frac{1}{H}\left[-i \frac{\left(\Gamma_{d}+\Gamma_{b}\right)}{2} \pm \sqrt{m_{\mathrm{eff}}^{2}-\frac{\left(3 H+\Gamma_{d}+\Gamma_{b}\right)^{2}}{4}}\right] .
$$

These eigenvalues show that the time evolution of the bulk inflaton field evaluated on the brane at late times is identical to that of a 4-dimensional scalar field satisfying the equation obtained by replacing $\Gamma_{\text {esc }}$ in Eq. (15) with $\Gamma_{\text {tot }}=\Gamma_{\text {esc }}+\Gamma_{d}$ $+\Gamma_{b}$. Here we have added $\Gamma_{\text {esc }}$ which comes from higher order corrections neglected in Eq. (27). This result shows that all three decay channels damp the oscillation of the bulk inflaton field as is expected.

So far we have studied just two specific examples. In more general cases, the eigenfunction cannot be constructed analytically, but we can justify the extensive use of the above results by perturbatively evaluating the shift of this pair of first eigenvalues. We consider that the unperturbed eigenfunction $u_{p}(y)$ is given by that for $U(y)=0$. Then applying the standard perturbation theory, we find that the shift of the eigenvalue $p^{2}$ due to the $U(y)$ term is given by

$$
\begin{aligned}
\Delta p^{2}= & -\left(i p+\frac{3}{2}\right) \int d y U(y)\left|u_{p}(y)\right|^{2} \\
& \approx \frac{-\left(i p+\frac{3}{2}\right)\left[\int_{0}^{\infty} d y \frac{R(y) \hat{\Gamma}_{b}(y)}{(y+H \ell)^{3}}+\frac{\Gamma_{d}}{2(H \ell)^{3}}\right]}{\int_{0}^{\infty} \frac{d y}{(y+H \ell)^{3}}}
\end{aligned}
$$

where we have used the fact that $\left|u_{p}(y)\right|^{2}$ behaves as $R(y)^{3} \approx[\ell /(|y|+H \ell)]^{3}$ near the brane. If we define $-(i p$ $+3 / 2) \Gamma_{b} H^{-1}$ by the right hand side of the above equation, we recover the same results obtained above in the analytically solvable cases. From the above equation, we also find that the dissipation is more efficient near the brane due to the effect of the warp factor. This fact implies that the energy transferred to the other bulk fields is also possibly trapped around the brane as in the case of the $\phi$ field. Of course, however, if the bulk fields do not have degrees of freedom localized near the brane, the energy density near the brane will soon vanish.

Now let us consider the cosmic expansion law in the present case. We divide $T_{a b}$ into two parts as

$$
T_{a b}=T_{a b}^{(\phi)}+T_{a b}^{(*)} .
$$

Here $T_{a b}^{(*)}$ is the energy-momentum tensor of the other bulk fields, into which the bulk inflaton field $\phi$ may decay. Then, the formulas for the energy density evolution of the matter fields on the brane (6) and that of the bulk fields (8) reduce to

$$
\begin{aligned}
\dot{\rho}+4 H \rho+H & \tau_{\mu}^{\mu}=\left[\Gamma_{d} \dot{\Phi}^{2}+2 T_{a b}^{(*)} u^{a} n^{b}\right]_{y=0}, \\
\dot{\rho}_{\mathrm{eff}}+4 H \rho_{\mathrm{eff}}= & {\left[-H\left(\dot{\Phi}^{2}-2 m_{\mathrm{eff}}^{2} \Phi^{2}\right)-\Gamma_{d} \dot{\Phi}^{2}\right.} \\
& \left.-2 H \ell T_{a b}^{(*)} n^{a} n^{b}\right]_{y=0},
\end{aligned}
$$

where we used $\Phi$ defined by Eq. (17). The cosmic expansion law depends on the nature of the fields composing $T_{a b}^{(*)}$. Below we discuss various cases one by one.

$$
\text { 1. }\left.T_{a b}^{(*)}\right|_{y=0} \approx 0
$$

Let us begin with the simplest situation that the bulk excitations caused by the decay of the $\phi$ field are not trapped around the brane. In this case $\left.T_{a b}^{(*)}\right|_{y=0}$ will rapidly diminish, 
and hence Eqs. (33) and (34) become almost identical to those in the case without dissipation in the bulk. The main effect of $\Gamma_{b}$ arises in $\Gamma_{\text {tot }}$, and it can be absorbed by the replacement of $\Gamma_{\text {esc }}$ with $\Gamma_{\text {esc }}+\Gamma_{b}$. Therefore we can immediately interpret that the $\Phi$-field decays into the dark radiation with the decay rate $\Gamma_{\mathrm{esc}}+\Gamma_{b}$ and into the matter fields on the brane with the decay rate $\Gamma_{d}$. If the matter fields on the brane stay radiationlike, the ratio of the dark radiation compared to the ordinary radiation is given by $\left(\Gamma_{\text {esc }}\right.$ $\left.+\Gamma_{b}\right) / \Gamma_{d}$. This ratio is constrained by the nucleosynthesis bound. If $\rho$ decreases slower like dust matter or cosmological constant for a certain period, the constraint by the nucleosynthesis becomes weaker.

$$
\text { 2. }\left.T_{a b}^{(*)}\right|_{y=0} \approx 0 \text { but }\left.T_{a b}^{(*)} u^{a} n^{b}\right|_{y=0}=0
$$

Next we consider the case that a part of resulting bulk excitation is trapped around the brane. Then, $\left.T_{a b}^{(*)}\right|_{y=0}$ will not diminish rapidly. Even in this case, if there is no direct interaction between the fields on the brane and the bulk fields other than $\phi$, we can set $\left.T_{a b}^{(*)} u^{a} n^{b}\right|_{y=0}=0$, since this term represents the energy transfer between them. Therefore the effect of nonvanishing $\left.T_{a b}^{(*)}\right|_{y=0}$ arises only in Eq. (34). Here the model qualitatively bifurcates into two cases, depending on whether this bulk excitation is completely trapped or not.

In the former case, the energy density of the trapped excitation does not decay into anything. We cannot further analyze the evolution of this energy density without specifying the details of the model. In the latter case, the energy of the trapped excitation eventually escapes to the infinity, and as a result $\left.T_{a b}^{(*)} n^{a} n^{b}\right|_{y=0}$ vanishes in the end. As in the case of the $\phi$ field discussed in the preceding section, vanishing of $\left.T_{a b}^{(*)} n^{a} n^{b}\right|_{y=0}$ will be interpreted as the transfer of the energy stored in the bulk modes into the dark radiation. As before, since the details of the model are not provided, we cannot analyze the evolution of the energy density of this trapped excitation before it is transferred into the dark radiation.

As a concrete example, let us consider the case that the decay product in the bulk is composed of the first pair of eigenmodes of another bulk scalar field. Then, since the effective energy density of this mode $\rho^{(*)}$ behaves like dust matter as in the case of the $\phi$ field, this field tends to dominate the energy density as a result of the cosmic expansion if the fields on the brane behave as radiation. Therefore, models with bulk fields of this type will produce large amount of dark matter, which is possibly transferred to dark radiation, and hence will be strongly constrained by the nucleosynthesis bound.

$$
\text { 3. } \left.\left.T_{a b}^{(*)}\right|_{y=0} \neq 0 \text { and }\left.T_{a b}^{(*)} u^{a} n^{b}\right|_{y=0}\right] \approx 0
$$

Finally, we consider the case that there is bulk excitation trapped near the brane and it has direct interaction with the fields on the brane. In such models, most of the discussions given in the previous case apply in the same way. However, the history of the universe can be quite different, if this interaction is sufficiently strong. In this case, the trapped excitation can efficiently decay into the ordinary matter fields on the brane. Then, the fraction of dark component is reduced, and hence such models can avoid the conflict with the nucleosynthesis bound.

\section{SUMMARY}

We investigated braneworld models of Randall-Sundrum type with a massive bulk scalar field, which we call the bulk inflaton here, taking into account various decay channels. We focused on how the cosmic expansion law realized on the brane is affected by the influence of the oscillating bulk inflaton and its decay products. The cosmic expansion law depends not only on the energy-momentum tensor of the fields localized on the brane but also on that of the bulk fields. Hence, in order to determine the cosmic expansion, we investigated the dynamics of the bulk inflaton including dissipation to the matter fields on the brane and in the bulk by using the Green function method developed in Refs. $[7,9,10]$.

It was argued that the effect of the bulk inflaton on the cosmic expansion can be mimicked by an effective 4-dimensional inflaton as long as $H^{2} \ell^{2} \ll 1$ and $\left|m^{2}\right| \ell^{2} \ll 1$ in Ref. [7]. Examining the late-time behavior of solutions of the bulk scalar field, we have shown that this claim is valid even if the bulk inflaton has various decay channels. The decay of the bulk inflaton occurs not only due to the dissipation to other fields on the brane or in the bulk but also due to the escape of energy to the direction away from the brane. From the analysis of the cosmic expansion law, we have shown that the decay product of this latter intrinsic dissipation should be interpreted as the dark radiation from the viewpoint of 4-dimensional effective theory. Further, we stress that this dark radiation is not identical to the contribution from the projected 5-dimensional Weyl tensor.

As for the dissipation due to interaction with matter fields on the brane, it has been already shown in Ref. [9] that the energy lost by this decay process can be interpreted as simply transferred to the matter fields on the brane, which is analogous to the usual decay process of the 4-dimensional inflaton field. The consequence of the decay due to interaction with other bulk fields has vast varieties. If the excitation generated by this decay process is not trapped near the brane at all, the effect is equivalent to the case of intrinsic dissipation. However, we also found that the dissipation to the bulk is more efficient near the brane. Hence, it is likely that a part of the lost energy is transferred to a mode localized near the brane. In this case, the decay product rather behaves as a 4-dimensional field as in the case of the bulk inflaton. The effective equation of state of such excitation measured through the cosmic expansion law depends on the details of the model setup, but at least we know that the effective equation of state is dustlike if the decay product consists of another massive scalar field. If the equation of state is dustlike, the fraction of the energy density of this excitation continues to increase during the radiation dominant phase. In the case that this excitation does not decay into the fields on the brane, it behaves as "dark matter." If this excitation is not completely trapped in the neighborhood of the brane, it finally decays into the dark radiation in the same way as we have seen for the bulk inflaton. This further decay transfers 
the energy from this "dark matter" to the dark radiation, but the dark component anyway in total dominates the energy density unless the decay rate of this excitation is sufficiently high or its initial amplitude is extremely small. Hence, such kind of models will be severely constrained by the requirement of the big-bang nucleosynthesis.

If the excitation in the bulk generated by the bulk inflaton decay has interaction with the fields on the brane, the energy can be transferred to the ordinary radiation fluid. If such decay process is efficient enough compared to the other decay channels, the final fraction of the dark component will not be significantly large. Such kind of models will be viable.

In conclusion, we found that the bulk inflaton model can reproduce the standard cosmology, but the branching ratio of the bulk inflaton decay and the nature of the decay products are constrained. The bulk inflaton model might leave observable signatures of the braneworld, and it may also provide a key to understanding the origin of dark matter.

\section{ACKNOWLEDGMENTS}

To complete this work, the discussion during and after the YITP workshops YITP-W-01-15 and YITP-W-02-19 was useful. This work was supported in part by the Monbukagakusho Grant-in-Aid Under Grants Nos. 14740165 and 14047212 .
[1] P. Horava and E. Witten, Nucl. Phys. B460, 506 (1996); B475, 94 (1996); N. Arkani-Hamed, S. Dimopoulos, and G. Dvali, Phys. Lett. B 429, 263 (1998); I. Antoniadis, N. ArkaniHamed, S. Dimopoulos, and G. Dvali, ibid. 436, 257 (1998); L. Randall and R. Sundrum, Phys. Rev. Lett. 83, 3370 (1999).

[2] L. Randall and R. Sundrum, Phys. Rev. Lett. 83, 4690 (1999).

[3] T. Shiromizu, K. Maeda, and M. Sasaki, Phys. Rev. D 62, 024012 (2000).

[4] J. Garriga and M. Sasaki, Phys. Rev. D 62, 043523 (2000).

[5] J. Garriga and T. Tanaka, Phys. Rev. Lett. 84, 2778 (2000); S.B. Giddings, E. Katz, and L. Randall, J. High Energy Phys. 03, 023 (2000).

[6] S. Mukohyama, Phys. Lett. B 473, 241 (2000); D. Ida, J. High Energy Phys. 09, 014 (2000); H. Stoica, S.H. Tye, and I. Wasserman, Phys. Lett. B 482, 205 (2000); H. Kodama, A. Ishibashi, and O. Seto, Phys. Rev. D 62, 064022 (2000); P. Binetruy, C. Deffayet, U. Ellwanger, and D. Langlois, Phys. Lett. B 477, 285 (2000); H.A. Bridgman, K.A. Malik, and D. Wands, Phys. Rev. D 65, 043502 (2002); D. Langlois and M. Rodriguez-Martinez, ibid. 64, 123507 (2001); K. Koyama, J. Soda, and S. Mukohyama, Phys. Lett. B 473, 241 (2000); Phys. Rev. D 62, 123502 (2000); K. Koyama and J. Soda, ibid. 65, 023514 (2002); E.E. Flanagan, S.H. Tye, and I. Wasser- man, Phys. Lett. B 522, 155 (2001); R. Maartens, Phys. Rev. D 62, 084023 (2000); C. Gordon and R. Maartens, ibid. 63, 044022 (2001); D. Langlois, R. Maartens, M. Sasaki, and D. Wands, ibid. 63, 084009 (2001).

[7] Y. Himemoto, T. Tanaka, and M. Sasaki, Phys. Rev. D 65, 104020 (2002).

[8] Y. Himemoto and M. Sasaki, Phys. Rev. D 63, 044015 (2001); N. Sago, Y. Himemoto, and M. Sasaki, ibid. 65, 024014 (2002); J. Yokoyama and Y. Himemoto, ibid. 64, 083511 (2001); S. Kobayashi, K. Koyama, and J. Soda, Phys. Lett. B 501, 157 (2001).

[9] Y. Himemoto and T. Tanaka, Phys. Rev. D 67, 084014 (2003).

[10] S.L. Dubovsky, V.A. Rubakov, and P.G. Tinyakov, Phys. Rev. D 62, 105011 (2000).

[11] C. van de Bruck, M. Dorca, C.J. Martins, and M. Parry, Phys. Lett. B 495, 183 (2000); P. Brax, C. van de Bruck, and A.C. Davis, J. High Energy Phys. 10, 026 (2001); E. Kiritsis, G. Kofinas, N. Tetradis, T.N. Tomaras, and V. Zarikas, ibid. 02, 035 (2003).

[12] A. Hebecker and J. March-Russell, Nucl. Phys. B608, 375 (2001); D. Langlois, L. Sorbo, and M. Rodriguez-Martinez, Phys. Rev. Lett. 89, 171301 (2002). 